\title{
Tips on Writing and Reviewing Articles as a Non-English Speaking Medical Doctor
}

\author{
Sun-Young Lee \\ Department of Internal Medicine, Konkuk University School of Medicine, Seoul, Korea
}

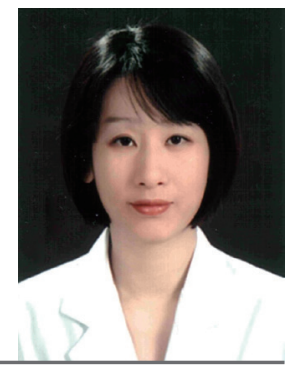

Writing and reviewing a scientific article is fairly time-consuming. One should become familiar with the required contents of an original article by reading previously published papers $[1,2]$. As a reviewer, you should not tell other people that you are reviewing the paper. You may not contact the author in person. After the completion of the review process, you must delete all of the files pertaining to the paper that you received. The reviewer is not allowed to manipulate the author's electronic document file. If you have any particular question for the authors about the paper, you should contact the journal editor and to send the inquiries to the authors. A good reviewer is someone who unveils the hidden potential of a study and helps the authors to present it in the most effective way.

Reviewing a scientific paper is a good way for specialists to update their knowledge in a particular area. Remember that journal editors want the reviewers to do two important things: 1) give comments on whether or not the paper should be published and 2) strengthen the paper before publication by asking the authors to revise their draft. If the study has nonremediable errors, then the paper should be rejected. Do not spend your time detecting typographical or grammatical errors. Provide a brief note about your final decision regarding whether or not the paper should be accepted in the "Comments to the editor" which is not sent to the authors. At this point, you can add other confidential details such as ethical issues found in the study, conflicts of interest, possible plagiarism, redundant duplication, and commercial affiliations. It is better to write the "Comments to the authors" in three sections: 1) summary, 2) major comments, and 3) minor comments (Table 1). The strengths and weaknesses of the Introduction, Methods, Results, and Discus- sion should be included in the major comments section.

The title should be related to the main topic of the study and be free of errors. The easiest ways to make the title attractive are to 1) mention the study design or the subjects, 2) refer to the study aim in the form of a question, and 3) summarize the main findings of the study. The title should not contain vague terms, irrational words, or abbreviations, with the exception of those approved by the specific journal to which the article is being submitted. It is wise to avoid starting the title with generic phrases such as "A study of", "A case of", or "Analysis of".

The Abstract is the most important part of a paper after the title, and in an original article, it comprises four sections: 1) Background/Aims, 2) Methods, 3) Results, and 4) Conclusions. The present tense is allowed only in the Background and Con-

\begin{tabular}{|c|c|}
\hline \multicolumn{2}{|l|}{ Comments to the editor } \\
\hline Confidential comments & $\begin{array}{l}\text { Whether or not the paper should be published } \\
\text { Ethical issues found in the study, conflicts of } \\
\text { interest, possible plagiarism, redundant } \\
\text { duplication, commercial affiliations, etc. }\end{array}$ \\
\hline \multicolumn{2}{|l|}{ Comments to the author } \\
\hline Summary & Brief summary of the manuscript \\
\hline Major comments & $\begin{array}{l}\text { Strengths and weaknesses of each section } \\
\text { Title and Abstract } \\
\text { Introduction } \\
\text { Methods } \\
\text { Results } \\
\text { Discussion } \\
\text { References } \\
\text { Tables and figures }\end{array}$ \\
\hline Minor comments & English (i.e., typographical or grammatical errors) \\
\hline
\end{tabular}


clusions, while the past tense is used for the Methods, Results, and Conclusions; thus, the Conclusions of the Abstract may be written either in the present or past tense, depending upon the specific journal style. The Background/Aims should use two or three short sentences to describe what is already known about the topic under study and why the study was conducted. Do not repeat the title or copy text from previous papers, include information that can be regarded as common sense, or define words like in a dictionary. In the Methods, use three of four sentences to describe how you performed the study, and use another three or four sentences in the Results to describe what you found. Use actual data and P-values to support your results. In the Conclusions, use one to three sentences to describe what the reported result means. Terms such as "might", "seem", and "could" are allowed in the Conclusions; however, there should be no discussion of either the work of other's or of your findings in the Abstract. All Abstract sections should be written as main body text, with no descriptions of references, figures, or tables.

The main body of the article generally comprises the Introduction, Methods, Results, Discussion, References, tables, and figure legends. The Introduction should contain at least one widely known fact pertaining to the study topic. Summarize the wellknown findings about the topic in the first paragraph, and then describe any unproven findings in the second paragraph. In the final paragraph of the Introduction, list your research questions, hypothesis, and study aims. The Methods should consist of descriptions of the main tools used in this study, including the statistical analysis. If it is a prospective study, the methodology could be further strengthened by describing how the optimum sample size was calculated. Do not forget to include any patient informed consents and institutional review board permissions in the Methods. The Results should describe the findings of the study in the same order as for the tools used to obtain them in the Methods, by citing actual data, P-values, tables, and figures. The Discussion should include a brief description of the relevant study findings in each paragraph, and should compare these findings with other published works. If there is a discrepancy, potential explanations thereof should be given. The last paragraph of the Discussion should provide the main conclusion of the study. About 20 references are recommended for an original article. When adding the references, be sure to include papers that can be searched for and which have an English-language abstract. The corresponding authors of these references could be used as preferred reviewers when you submit your paper.

With regard to the use of English in the paper, numbers less than or equal ten should be written out in full. The numeral should be used for all numbers above ten, except at the beginning of a sentence where they should be written out in full.
"Data" is plural, and therefore should be used in conjunction with "are" or "were". Exclamation marks and contractions such as "didn't", "haven't", and "I'm" should not be used. When appearing at the start of a sentence, "And" should be changed to "In addition", and "But" should be changed to "However". "A" should be used for indefinite articles (i.e., a nonspecific noun), and "the" for definite articles (i.e., a specific noun). When searching for a "free grammar check" using an Internet search engine, please do not forget to change the interface language to English and to change your location as appropriate referring to the type of English (American or British).

Journal publications are more interesting when one understands about the journal impact factor. The impact factor is calculated by dividing the number of citations (source+non-source items) by the total number of published source items. Since the source items consist of original articles, review articles, and case reports, the impact factor increases when the journal publishes updated review articles written by famous authors.

Another way to become interested in journal publications is to understand the trends of publication in a certain issue [35]. When a new therapeutic method is developed, the findings are usually submitted as a "Case report" or "Letter to the editor". "Original articles" then follow, and include many "me too" papers in a variety of journals, followed by review articles and meta-analyses. Widely-cited papers, so-called landmark papers, are usually published as original articles or review articles. Papers on therapeutic procedures with wider applications and easier techniques will be cited more often than those with narrower applications and difficult techniques. Similarly, papers that include results relevant to multiple journals and countries are usually cited more often than those relevant to only particular journals or countries.

Writing and reviewing in English is more difficult for scientists whose first language is not English. However, it cannot be avoided because medicine is a science that is continually and rapidly being updated in the English-language literature. Thus, these tips for writing and reviewing a scientific paper should prove helpful.

\section{REFERENCES}

1. Audisio RA, Stahel RA, Aapro MS, et al. Successful publishing: how to get your paper accepted. Surg Oncol 2009; 18:350-6.

2. Groves $T$. What makes a high quality clinical research paper? Oral Dis 2010;16:313-5.

3. Lee JH. Analysis of CrossCheck data on two years' worth of papers submitted to Archives of Plastic Surgery. Arch Plast 
Surg 2014;41:449-51.

4. Davis PM, Walters WH. The impact of free access to the scientific literature: a review of recent research. J Med Libr Assoc 2011;99:208-17.

5. Kim JT. Where is Archives of Plastic Surgery now? And where is it heading? Arch Plast Surg 2014;41:309-11.
Correspondence: Sun-Young Lee

Department of Internal Medicine, Konkuk University School of Medicine, 120-1 Neungdong-ro Gepartment of Internal Medicine, Kongjin-gu, Seoul 143-729, Korea

Tel: +82-2-2030-7747, Fax: +82-2-2030-7748, E-mail: sunyoung@ kuh.ac.kr

This work was supported by the National Research Foundation of Korea funded by the Korean Government (NRF 2012K2A2A4010622).

No potential conflict of interest relevant to this article was reported.

Received: 27 Nov $2014 \bullet$ Revised: 27 Nov $2014 \bullet$ Accepted: 27 Nov 2014 pISSN: 2234-6163 • elSSN: 2234-6171

http://dx.doi.org/10.5999/aps.2015.42.1.1 • Arch Plast Surg 2015;42:1-3 